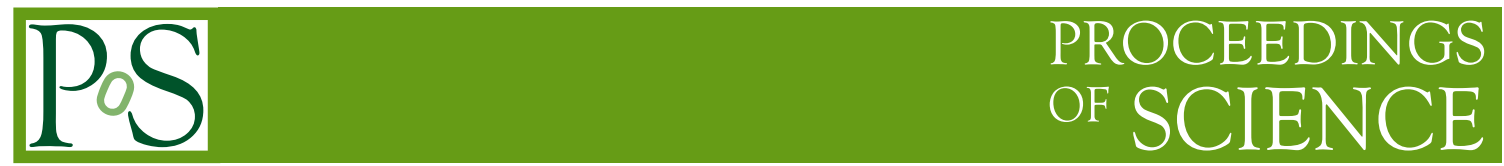

\title{
Appell-Lauricella's hypergeometric functions and intersection theory
}

\section{Yoshiaki Goto}

Otaru University of Commerce,

Midori 3-5-21, Otaru, Japan

E-mail: goto@res.otaru-uc.ac.jp

We study hypergeometric functions by applying the intersection theory for twisted homology and cohomology groups, which arise from their Euler-type integral representations.

In this article, we consider Appell-Lauricella's hypergeometric functions which are classical generalizations of Gauss' hypergeometric function. Especially, we focus on Lauricella's $F_{A}$ and $F_{C}$, and study them by using intersection theory. By evaluating some intersection numbers, we obtain quadratic relations between $F_{A}$ or $F_{C}$ as a consequence of the twisted period relations.

MathemAmplitudes 2019: Intersection Theory \& Feynman Integrals (MA2019)

18-20 December 2019

Padova, Italy 


\section{Introduction}

Gauss' hypergeometric function is defined by

$$
{ }_{2} F_{1}(a, b, c ; x)=\sum_{n=0}^{\infty} \frac{(a)_{n}(b)_{n}}{(c)_{n}(1)_{n}} x^{n},
$$

where $x \in \mathbb{C}$ is a variable, $a, b, c \in \mathbb{C}$ are parameters $\left(c \notin \mathbb{Z}_{\leq 0}\right)$, and we set $(\alpha)_{n}=\alpha(\alpha+1) \cdots(\alpha+$ $n-1)=\Gamma(\alpha+n) / \Gamma(\alpha)$. This series converges on $\{x \in \mathbb{C}|| x \mid<1\}$. It is well-known that ${ }_{2} F_{1}(a, b, c ; x)$ satisfies the hypergeometric differential equation

$$
\left(x(1-x) \frac{d^{2}}{d x^{2}}+(c-(a+b+1) x) \frac{d}{d x}-a b\right) f(x)=0,
$$

and that if $\operatorname{Re}(a), \operatorname{Re}(c-a)>0$, then ${ }_{2} F_{1}(a, b, c ; x)$ admits an Euler-type integral representation:

$$
{ }_{2} F_{1}(a, b, c ; x)=\frac{\Gamma(c)}{\Gamma(a) \Gamma(c-a)} \int_{0}^{1} t^{a}(1-t)^{c-a}(1-x t)^{-b} \frac{d t}{t(1-t)} .
$$

As classical generalizations of ${ }_{2} F_{1}$, Lauricella's multi-variable hypergeometric functions $F_{A}, F_{B}$, $F_{C}$ and $F_{D}$ are well-known. These are also called Appell's hypergeometric functions when they are in two variables.

The hypergeometric function ${ }_{2} F_{1}$ and its generalizations are studied from various view points. In this article, we study them by applying the intersection theory for twisted homology and cohomology groups. Twisted (co)homology groups are associated with local systems defined by multi-valued functions that are integrands of Euler-type integral representations. For example, when we consider ${ }_{2} F_{1}$, we use the local system defined by the multi-valued function $t^{a}(1-t)^{c-a}(1-x t)^{-b}$ in $t$ which appears in (1). Aomoto applied such homology and cohomology groups to study of hypergeometric functions.

Intersection pairings of (co)homology groups with coefficients in local systems were defined in [16]. However, since these definitions are written in terms of homological algebra, it seems not easy to evaluate the intersection numbers directly. By [9], intersection numbers of twisted homology groups can be evaluated in terms of topological intersection numbers and branches of the multi-valued function. By [2] and [12], intersection numbers of twisted cohomology groups are expressed by residues of logarithmic forms.

The intersection theory for twisted homology groups are applied to study of the monodromy representations, connection problems, and so on. That for twisted cohomology groups are applied to study of Pfaffian equations, contiguity relations, and so on. By the compatibility of these intersection pairings and the pairings between twisted homology and cohomology groups given by integrations, we can obtain the twisted period relations which imply quadratic relations between hypergeometric functions.

In [15] which is in the same proceedings, Matsumoto introduces the intersection theory for twisted (co)homology groups associated with multi-valued functions in one variable, which arise from ${ }_{2} F_{1}$ and Lauricella's $F_{D}$. In this article, we treat those associated with multi-valued functions in multi-variables, focusing on two examples arising from Lauricella's $F_{A}$ and $F_{C}$. 
This article is arranged as follows. In Section 2, we give the definitions of Lauricella's hypergeometric functions. We also introduce their Euler-type integral representations and the systems $E_{A}, E_{B}, E_{C}$ and $E_{D}$ of differential equations satisfied by $F_{A}, F_{B}, F_{C}$ and $F_{D}$, respectively. In Section 3, we give settings of twisted (co)homology groups and intersection pairings to study $F_{A}$ and $F_{C}$. In Section 4, we explain how to construct twisted cycles that corresponds to series solutions to $E_{A}$ or $E_{C}$. By evaluating intersection numbers, we obtain quadratic relations between $F_{A}$ or $F_{C}$ from the twisted period relations.

\section{Appell-Lauricella's hypergeometric functions}

In this section, we give the definitions of Appell-Lauricella's multi-variable hypergeometric functions, and collect some basic facts referring to [14].

\subsection{Lauricella's hypergeometric series}

Lauricella's hypergeometric functions of $m$ variables $x=\left(x_{1}, \ldots, x_{m}\right)$ are defined as follows:

$$
\begin{aligned}
& F_{A}\left(a, b_{1}, \ldots, b_{m}, c_{1}, \ldots, c_{m} ; x\right) \\
& =\sum_{n_{1}, \ldots, n_{m}=0}^{\infty} \frac{(a)_{n_{1}+\cdots+n_{m}}\left(b_{1}\right)_{n_{1}} \cdots\left(b_{m}\right)_{n_{m}}}{\left(c_{1}\right)_{n_{1}} \cdots\left(c_{m}\right)_{n_{m}} n_{1} ! \cdots n_{m} !} x_{1}^{n_{1}} \cdots x_{m}^{n_{m}} \quad\left(\left|x_{1}\right|+\cdots+\left|x_{m}\right|<1\right), \\
& F_{B}\left(a_{1}, \ldots, a_{m}, b_{1}, \ldots, b_{m}, c ; x\right) \\
& =\sum_{n_{1}, \ldots, n_{m}=0}^{\infty} \frac{\left(a_{1}\right)_{n_{1}} \cdots\left(a_{m}\right)_{n_{m}}\left(b_{1}\right)_{n_{1}} \cdots\left(b_{m}\right)_{n_{m}}}{(c)_{n_{1}+\cdots+n_{m}} n_{1} ! \cdots n_{m} !} x_{1}^{n_{1}} \cdots x_{m}^{n_{m}} \quad\left(\left|x_{1}\right|, \ldots,\left|x_{m}\right|<1\right), \\
& F_{C}\left(a, b, c_{1}, \ldots, c_{m} ; x\right) \\
& =\sum_{n_{1}, \ldots, n_{m}=0}^{\infty} \frac{(a)_{n_{1}+\cdots+n_{m}}(b)_{n_{1}+\cdots+n_{m}}}{\left(c_{1}\right)_{n_{1}} \cdots\left(c_{m}\right)_{n_{m}} n_{1} ! \cdots n_{m} !} x_{1}^{n_{1}} \cdots x_{m}^{n_{m}} \quad\left(\sqrt{\left|x_{1}\right|}+\cdots+\sqrt{\left|x_{m}\right|}<1\right), \\
& F_{D}\left(a, b_{1}, \ldots, b_{m}, c ; x\right) \\
& =\sum_{n_{1}, \ldots, n_{m}=0}^{\infty} \frac{(a)_{n_{1}+\cdots+n_{m}}\left(b_{1}\right)_{n_{1}} \cdots\left(b_{m}\right)_{n_{m}}}{(c)_{n_{1}+\cdots+n_{m}} n_{1} ! \cdots n_{m} !} x_{1}^{n_{1}} \cdots x_{m}^{n_{m}} \quad\left(\left|x_{1}\right|, \ldots,\left|x_{m}\right|<1\right) .
\end{aligned}
$$

When $m=2$, Lauricella's $F_{A}$ (resp. $F_{B}, F_{C}, F_{D}$ ) is also called Appell's hypergeometric function $F_{2}\left(\right.$ resp. $\left.F_{3}, F_{4}, F_{1}\right)$.

\begin{tabular}{|c||c|c|c|c|}
\hline Lauricella & $F_{A}$ & $F_{B}$ & $F_{C}$ & $F_{D}$ \\
\hline Appell & $F_{2}$ & $F_{3}$ & $F_{4}$ & $F_{1}$ \\
\hline
\end{tabular}

Table 1

\subsection{Euler-type integral representations}

To obtain Euler-type integral representations of Lauricella's hypergeometric functions, we use

$$
\int_{0}^{1} t^{p-1}(1-t)^{q-1} d t=B(p, q)=\frac{\Gamma(p) \Gamma(q)}{\Gamma(p+q)}
$$


and its generalization

$$
\int_{\Delta^{m}} \prod_{k=1}^{m} t_{k}^{p_{k}-1} \cdot\left(1-\sum_{k=1}^{m} t_{k}\right)^{p_{m+1}-1} d t_{1} \wedge \cdots \wedge d t_{m}=\frac{\prod_{k=1}^{m+1} \Gamma\left(p_{k}\right)}{\Gamma\left(\sum_{k=1}^{m+1} p_{k}\right)},
$$

where $\Delta^{m}=\left\{\left(t_{1}, \ldots, t_{m}\right) \in \mathbb{R}^{m} \mid t_{1}, \ldots, t_{m}, 1-\sum_{k=1}^{m} t_{k}>0\right\}$.

\subsubsection{Euler-type integral representation of $F_{A}$}

For simplicity, we denote $\sum=\sum_{k=1}^{m}$ and $\prod=\prod_{k=1}^{m}$. If $\operatorname{Re}\left(b_{k}\right), \operatorname{Re}\left(c_{k}-b_{k}\right)>0$, then

$$
\begin{aligned}
& F_{A}\left(a, b_{1}, \ldots, b_{m}, c_{1}, \ldots, c_{m} ; x\right) \\
& =\prod \frac{\Gamma\left(c_{k}\right)}{\Gamma\left(b_{k}\right) \Gamma\left(c_{k}-b_{k}\right)} \cdot \int_{(0,1)^{m}} \prod\left(t_{k}^{b_{k}-1} \cdot\left(1-t_{k}\right)^{c_{k}-b_{k}-1}\right) \cdot\left(1-\sum x_{k} t_{k}\right)^{-a} d t,
\end{aligned}
$$

where $d t=d t_{1} \wedge \cdots \wedge d t_{m}$. To show this expression, it is sufficient to consider the power series expansion of $\left(1-\sum x_{k} t_{k}\right)^{-a}$.

\subsubsection{Euler-type integral representation of $F_{B}$}

If $\operatorname{Re}\left(b_{k}\right), \operatorname{Re}\left(c-\sum b_{k}\right)>0$, then

$$
\begin{aligned}
& F_{B}\left(a_{1}, \ldots, a_{m}, b_{1}, \ldots, b_{m}, c ; x\right) \\
& =\frac{\Gamma(c)}{\Gamma\left(c-\sum b_{k}\right) \prod \Gamma\left(b_{k}\right)} \cdot \int_{\Delta^{m}} \prod\left(t_{k}^{b_{k}-1} \cdot\left(1-x_{k} t_{k}\right)^{-a_{k}}\right) \cdot\left(1-\sum t_{k}\right)^{c-\sum b_{k}-1} d t .
\end{aligned}
$$

If we put $t_{k}=\frac{s_{k}}{x_{k}}$, the differential form changes into

$$
\prod x_{k}^{-b_{k}} \cdot \prod\left(s_{k}^{b_{k}-1} \cdot\left(1-s_{k}\right)^{-a_{k}}\right) \cdot\left(1-\sum \frac{1}{x_{k}} s_{k}\right)^{c-\sum b_{k}-1} d s
$$

By the integral representation (2), we expect that $F_{B}\left(a_{1}, \ldots, a_{m}, b_{1}, \ldots, b_{m}, c ; x_{1}, \ldots, x_{m}\right)$ and

$$
\prod x_{k}^{-b_{k}} \cdot F_{A}\left(\sum b_{k}-c+1, b_{1}, \ldots, b_{m}, b_{1}-a_{1}+1, \ldots, b_{m}-a_{m}+1 ; \frac{1}{x_{1}}, \ldots, \frac{1}{x_{m}}\right) .
$$

have similar properties; see also Remark 2.1.

\subsubsection{Euler-type integral representation of $F_{C}$}

By considering the power series expansion, we expect the integral representation

$$
\begin{aligned}
& F_{C}\left(a, b, c_{1}, \ldots, c_{m} ; x\right) \\
& =\frac{\Gamma(1-a)}{\prod \Gamma\left(1-c_{k}\right) \cdot \Gamma\left(\sum c_{k}-a-m+1\right)} \cdot \int_{\Delta} \prod t_{k}^{-c_{k}} \cdot\left(1-\sum t_{k}\right)^{\sum c_{k}-a-m}\left(1-\sum \frac{x_{k}}{t_{k}}\right)^{-b} d t
\end{aligned}
$$

However, if we put $\Delta=\Delta^{m}$, the power series expansion of $\left(1-\sum \frac{x_{k}}{t_{k}}\right)^{-b}$ is divergent. A "twisted cycle" $\Delta$ such that this integral representation holds has been constructed in [8]. In Section 4.2, we will explain the construction. Note that the zero set of the factor $\left(1-\sum \frac{x_{k}}{t_{k}}\right)$ is not a hyperplane, while those of the factors in the integral representations of $F_{A}, F_{B}$ and $F_{D}$ are hyperplanes. 


\subsubsection{Euler-type integral representations of $F_{D}$}

There are two types of integral representations of $F_{D}$. The first one is expressed as a onedimensional integral; if $\operatorname{Re}(a), \operatorname{Re}(c-a)>0$, then

$$
F_{D}\left(a, b_{1}, \ldots, b_{m}, c ; x\right)=\frac{\Gamma(c)}{\Gamma(a) \Gamma(c-a)} \cdot \int_{0}^{1} t^{a-1}(1-t)^{c-a-1} \prod_{i=1}^{m}\left(1-x_{i} t\right)^{-b_{i}} d t .
$$

The second one is expressed as an $m$-dimensional integral; if $\operatorname{Re}\left(b_{k}\right), \operatorname{Re}\left(c-\sum b_{k}\right)>0$, then

$$
\begin{aligned}
& F_{D}\left(a, b_{1}, \ldots, b_{m}, c ; x\right) \\
& =\frac{\Gamma(c)}{\Gamma\left(c-\sum b_{k}\right) \prod \Gamma\left(b_{k}\right)} \cdot \int_{\Delta^{m}} \prod t_{k}^{b_{k}-1} \cdot\left(1-\sum t_{k}\right)^{c-\sum b_{k}-1}\left(1-\sum x_{k} t_{k}\right)^{-a} d t
\end{aligned}
$$

\subsection{Differential equations}

Lauricella's $F_{A}, F_{B}, F_{C}$ and $F_{D}$ are solutions to the regular holonomic systems $E_{A}, E_{B}, E_{C}$ and $E_{D}$ of linear differential equations, respectively.

\subsubsection{Differential equations for $F_{A}$}

We set $\partial_{i}=\frac{\partial}{\partial x_{i}}$. Lauricella's $F_{A}\left(a, b_{1}, \ldots, b_{m}, c_{1}, \ldots, c_{m} ; x\right)$ satisfies

$$
\left(x_{k}\left(1-x_{k}\right) \partial_{k}^{2}-x_{k} \sum_{\substack{1 \leq i \leq m \\ i \neq k}} x_{i} \partial_{k} \partial_{i}+\left(c_{k}-\left(a+b_{k}+1\right) x_{k}\right) \partial_{k}-b_{k} \sum_{\substack{1 \leq i \leq m \\ i \neq k}} x_{i} \partial_{i}-a b_{k}\right) f(x)=0
$$

for $k=1, \ldots, m$. Let $E_{A}\left(a, b_{1}, \ldots, b_{m}, c_{1}, \ldots, c_{m}\right)$ be the system of differential equations generated by them. It is known that the rank of $E_{A}$ is $2^{m}$, and the set of singular points (called the singular locus) is

$$
S_{A}=\left(\prod_{k=1}^{m} x_{k} \cdot \prod_{\left\{i_{1}, \ldots, i_{r}\right\} \subset\{1, \ldots, m\}}\left(1-\sum_{p=1}^{r} x_{i_{p}}\right)=0\right) \subset \mathbb{C}^{m} .
$$

For example, $S_{A}=\left(x_{1} x_{2}\left(1-x_{1}\right)\left(1-x_{2}\right)\left(1-x_{1}-x_{2}\right)=0\right)$ if $m=2$. Let $x \notin S_{A}$ be a point near to the origin. If $c_{k} \notin \mathbb{Z}$, then a basis of the local solution space of $E_{A}$ is given by

$$
f_{A}^{i_{1} \cdots i_{r}}=\prod_{p=1}^{r} x_{i_{p}}^{1-c_{i_{p}}} \cdot F_{A}\left(a+r-\sum_{p=1}^{r} c_{i_{p}}, b^{I}, c^{I} ; x\right) \quad\left(I=\left\{i_{1}, \ldots, i_{r}\right\} \subset\{1, \ldots, m\}\right),
$$

where the vectors $b^{I}=\left(b_{1}^{I}, \ldots, b_{m}^{I}\right)$ and $c^{I}=\left(c_{1}^{I}, \ldots, c_{m}^{I}\right)$ are defined by

$$
\left\{\begin{array} { l } 
{ b _ { i _ { p } } ^ { I } = b _ { i _ { p } } + 1 - c _ { i _ { p } } , } \\
{ b _ { j } ^ { I } = b _ { j } , }
\end{array} \quad \left\{\begin{array}{l}
c_{i_{p}}^{I}=2-c_{i_{p}}, \\
c_{j}^{I}=c_{j}
\end{array} \quad\left(i_{p} \in I, j \notin I\right) .\right.\right.
$$

Note that if $I=\emptyset$, then $f_{A}^{\emptyset}=F_{A}\left(a, b_{1}, \ldots, b_{m}, c_{1}, \ldots, c_{m} ; x\right)$. 


\subsubsection{Differential equations for $F_{B}$}

Lauricella's $F_{B}\left(a_{1}, \ldots, a_{m}, b_{1}, \ldots, b_{m}, c ; x\right)$ satisfies

$$
\left(x_{k}\left(1-x_{k}\right) \partial_{k}^{2}+\sum_{\substack{1 \leq i \leq m \\ i \neq k}} x_{i} \partial_{k} \partial_{i}+\left(c-\left(a_{k}+b_{k}+1\right) x_{k}\right) \partial_{k}-a_{k} b_{k}\right) f(x)=0
$$

for $k=1, \ldots, m$. Let $E_{B}\left(a_{1}, \ldots, a_{m}, b_{1}, \ldots, b_{m}, c\right)$ be the system of differential equations generated by them.

Remark 2.1. In fact, by setting $x_{k}=\frac{1}{\xi_{k}}(k=1, \ldots, m)$, we have

$f(x)$ is a solution to $E_{B}\left(a_{1}, \ldots, a_{m}, b_{1}, \ldots, b_{m}, c\right)$

$\Longleftrightarrow \prod \xi^{b_{k}} \cdot f(\xi)$ is a solution to $E_{A}\left(\sum b_{k}-c+1, b_{1}, \ldots, b_{m}, b_{1}-a_{1}+1, \ldots, b_{m}-a_{m}+1\right)$.

Thus, some results for $F_{B}$ are obtained from those for $F_{A}$.

\subsubsection{Differential equations for $F_{C}$}

Lauricella's $F_{C}\left(a, b, c_{1}, \ldots, c_{m} ; x\right)$ satisfies

$$
\begin{array}{r}
\left(x_{k}\left(1-x_{k}\right) \partial_{k}^{2}-x_{k} \sum_{\substack{1 \leq i \leq m \\
i \neq k}} x_{i} \partial_{i} \partial_{k}-\sum_{\substack{1 \leq i, j \leq m \\
i \neq k}} x_{i} x_{j} \partial_{i} \partial_{j}+\left(c_{k}-(a+b+1) x_{k}\right) \partial_{k}\right. \\
\left.-(a+b+1) \sum_{\substack{1 \leq i \leq m \\
i \neq k}} x_{i} \partial_{i}-a b\right) f(x)=0
\end{array}
$$

for $k=1, \ldots, m$. Let $E_{C}\left(a, b, c_{1}, \ldots, c_{m}\right)$ be the system of differential equations generated by them. It is known that the rank of $E_{C}$ is $2^{m}$, and the singular locus is

$$
S_{C}=\left(\prod_{k=1}^{m} x_{k} \cdot \prod_{\varepsilon_{1}, \ldots \varepsilon_{m}= \pm 1}\left(1+\sum_{k} \varepsilon_{k} \sqrt{x_{k}}\right)=0\right) \subset \mathbb{C}^{m}
$$

For example, $S_{C}=\left(x_{1} x_{2}\left(x_{1}^{2}+x_{2}^{2}-2 x_{1} x_{2}-2 x_{1}-2 x_{2}+1\right)=0\right)$ if $m=2$. Let $x \notin S_{C}$ be a point near to the origin. If $c_{k} \notin \mathbb{Z}$, then a basis of the local solution space of $E_{C}$ is given by

$$
f_{C}^{i_{1} \cdots i_{r}}=\prod_{p=1}^{r} x_{i_{p}}^{1-c_{i_{p}}} \cdot F_{C}\left(a+r-\sum_{p=1}^{r} c_{i_{p}}, b+r-\sum_{p=1}^{r} c_{i_{p}}, c^{I} ; x\right) \quad\left(I=\left\{i_{1}, \ldots, i_{r}\right\} \subset\{1, \ldots, m\}\right),
$$

where the vector $c^{I}=\left(c_{1}^{I}, \ldots, c_{m}^{I}\right)$ is defined by

$$
\begin{cases}c_{i_{p}}^{I}=2-c_{i_{p}} & \left(i_{p} \in I\right) \\ c_{j}^{I}=c_{j} & (j \notin I)\end{cases}
$$

Note that if $I=\emptyset$, then $f_{C}^{\emptyset}=F_{C}\left(a, b, c_{1}, \ldots, c_{m} ; x\right)$. 


\subsubsection{Differential equations for $F_{D}$}

Lauricella's $F_{D}\left(a, b_{1}, \ldots, b_{m}, c ; x\right)$ satisfies

$$
\left(x_{k}\left(1-x_{k}\right) \partial_{k}^{2}+\left(1-x_{k}\right) \sum_{\substack{1 \leq i \leq m \\ i \neq k}} x_{i} \partial_{i} \partial_{k}+\left(c-\left(a+b_{k}+1\right) x_{k}\right) \partial_{k}-b_{k} \sum_{\substack{1 \leq i \leq m \\ i \neq k}} x_{i} \partial_{i}-a b_{k}\right) f(x)=0
$$

for $k=1, \ldots, m$, and

$$
\left(\left(x_{i}-x_{j}\right) \partial_{i} \partial_{j}-b_{j} \partial_{i}+b_{i} \partial_{j}\right) f(x)=0
$$

for $1 \leq i<j \leq m$. Let $E_{D}\left(a, b_{1}, \ldots, b_{m}, c\right)$ be the system of differential equations generated by them. It is known that the rank of $E_{D}$ is $m+1$, and the singular locus is

$$
S_{D}=\left(\prod_{k=1}^{m} x_{k}\left(1-x_{k}\right) \cdot \prod_{1 \leq i<j \leq m}\left(x_{i}-x_{j}\right)=0\right) \subset \mathbb{C}^{m} .
$$

For example, $S_{D}=\left(x_{1} x_{2}\left(1-x_{1}\right)\left(1-x_{2}\right)\left(x_{1}-x_{2}\right)=0\right)$ if $m=2$.

We do not have a basis of the local solution space, which are expressed by $F_{D}$. On the other hand, $F_{D}$ is well-studied from the view point of the integral representation (4), because twisted (co)homology theory for a one-dimensional integral is easier than the higher dimensional cases. In [15], the study of $F_{D}$ in the framework of the intersection theory for twisted (co)homology groups is explained.

\section{Twisted homology and cohomology groups for $F_{A}$ and $F_{C}$}

In this article, we focus on $F_{A}$ and $F_{C}$. We study them by using twisted (co)homology groups that are associated with the Euler-type integral representations.

Assumption 3.1. Hereafter, we assume that the parameters satisfy some non-integral conditions.

- For $F_{A}$, we assume

$$
\begin{aligned}
& b_{1}, \ldots, b_{m}, c_{1}-b_{1}, \ldots, c_{m}-b_{m}, a-\sum_{p=1}^{r} c_{i_{r}} \notin \mathbb{Z} \quad \text { (for any }\left\{i_{1}, \ldots, i_{r}\right\} \subset\{1, \ldots, m\} \text { ), } \\
& c_{1}, \ldots, c_{m} \notin \mathbb{Z} .
\end{aligned}
$$

- For $F_{C}$, we assume

$$
\begin{aligned}
& \left.a-\sum_{p=1}^{r} c_{i_{r}}, \quad b-\sum_{p=1}^{r} c_{i_{r}} \notin \mathbb{Z} \quad \text { (for any }\left\{i_{1}, \ldots, i_{r}\right\} \subset\{1, \ldots, m\}\right), \\
& c_{1}, \ldots, c_{m} \notin \mathbb{Z} .
\end{aligned}
$$

The system $E_{A}$ (resp. $E_{C}$ ) is irreducible when the condition (7) (resp. (9)) holds. Under the condition (8) (resp. (10)), the series solutions (5) (resp. (6)) form a basis of local solution space of $E_{A}\left(\right.$ resp. $\left.E_{C}\right)$.

For basic ideas of twisted (co)homology groups and intersection theory, refer to [15]. 


\subsection{Twisted homology and cohomology groups}

Recall the integral representations (2) and (3). Up to $\Gamma$-factors, $F_{A}$ and $F_{C}$ are expressed by the integrals

$$
\begin{aligned}
& \int_{(0,1)^{m}} \prod\left(t_{k}^{b_{k}}\left(1-t_{k}\right)^{c_{k}-b_{k}-1}\right) \cdot\left(1-\sum x_{k} t_{k}\right)^{-a} \frac{d t}{\prod t_{k}} \\
& \int_{\Delta} \prod t_{k}^{1-c_{k}+b} \cdot\left(1-\sum t_{k}\right)^{\sum c_{k}-a-m+1} \cdot w_{x}(t)^{-b} \frac{d t}{\prod t_{k}\left(1-\sum t_{k}\right)},
\end{aligned}
$$

respectively, where we set $w_{x}(t)=\prod t_{k} \cdot\left(1-\sum \frac{x_{k}}{t_{k}}\right)$ which is a polynomial of degree $m$. Let us consider twisted (co)homology theory for the multi-valued functions

$$
\begin{aligned}
& U_{A}(t)=\prod\left(t_{k}^{b_{k}}\left(1-t_{k}\right)^{c_{k}-b_{k}-1}\right) \cdot\left(1-\sum x_{k} t_{k}\right)^{-a}, \\
& U_{C}(t)=\prod t_{k}^{1-c_{k}+b} \cdot\left(1-\sum t_{k}\right)^{\sum c_{k}-a-m+1} \cdot w_{x}(t)^{-b}
\end{aligned}
$$

defined on

$$
\begin{aligned}
& T_{A}=\mathbb{C}^{m}-\left(\bigcup\left(t_{k}=0\right) \cup \bigcup\left(1-t_{k}=0\right) \cup\left(1-\sum x_{k} t_{k}=0\right)\right), \\
& T_{C}=\mathbb{C}^{m}-\left(\bigcup\left(t_{k}=0\right) \cup\left(1-\sum t_{k}=0\right) \cup\left(w_{x}(t)=0\right)\right),
\end{aligned}
$$

respectively. Here, we fix $x=\left(x_{1}, \ldots, x_{m}\right)$ and regard $t=\left(t_{1}, \ldots, t_{m}\right)$ as variables.

Remark 3.2. In (11) and (12), we take out the differential forms $\frac{d t}{\prod t_{k}}$ and $\frac{d t}{\prod t_{k}\left(1-\sum t_{k}\right)}$, respectively. If we choose other differential forms, structures of twisted cohomology groups are slightly changed. However, it is not essential in this article.

\subsubsection{Twisted homology groups}

Let \# be $A$ or $C$. We set

$$
\begin{aligned}
& \mathcal{C}_{k}=\left\{\sum_{j: \text { finite }} a_{j} \cdot \Delta_{j} \otimes U_{\#, \Delta_{j}} \mid a_{j} \in \mathbb{C}, \Delta_{j}: k \text {-simplex }\right\}, \\
& C_{k}^{\mathrm{lf}}=\left\{\sum_{j: \text { locally finite }} a_{j} \cdot \Delta_{j} \otimes U_{\#, \Delta_{j}} \mid a_{j} \in \mathbb{C}, \Delta_{j}: k \text {-simplex }\right\} \supset C_{k},
\end{aligned}
$$

where $\Delta \otimes U_{\#, \Delta}$ denotes the pair of the simplex $\Delta \subset T_{\#}$ and the branch $U_{\#, \Delta}(t)$ of $U_{\#}(t)$ on $\Delta$. We define twisted boundary operators $\partial^{U_{\#}}: C_{k} \rightarrow C_{k-1}$ and $\partial^{U_{\sharp}}: C_{k}^{\text {lf }} \rightarrow C_{k-1}^{\text {lf }}$ by

$$
\partial^{U_{\#}}\left(\Delta \otimes U_{\#, \Delta}\right)=\left.\partial \Delta \otimes U_{\#, \Delta}\right|_{\partial \Delta} .
$$

Since we have $\partial^{U_{\#}} \circ \partial^{U_{\#}}=0$, we define the $k$-th twisted homology group and the $k$-th locally finite twisted homology group by

$H_{k}\left(T_{\#}, U_{\#}\right)=\operatorname{ker}\left(\partial^{U_{\#}}: C_{k} \rightarrow C_{k-1}\right) / \partial^{U_{\#}}\left(C_{k+1}\right), \quad H_{k}^{\mathrm{lf}}\left(T_{\#}, U_{\#}\right)=\operatorname{ker}\left(\partial^{U_{\#}}: C_{k}^{\mathrm{lf}} \rightarrow C_{k-1}^{\mathrm{lf}}\right) / \partial^{U_{\#}}\left(C_{k+1}^{\mathrm{lf}}\right)$, respectively. An element of $\operatorname{ker}\left(\partial^{U_{\#}}\right)$ is called a twisted cycle. 


\subsubsection{Twisted cohomology groups}

Let $\Omega^{k}\left(T_{\#}\right)$ be the space of the rational $k$-forms on $\mathbb{P}^{k}$ that have poles along $\mathbb{P}^{k}-T_{\#}$. We set

$$
\omega_{\#}=\frac{d U_{\#}}{U_{\#}} \in \Omega^{1}\left(T_{\#}\right) .
$$

Since $\nabla_{\#}=d+\omega_{\#} \wedge: \Omega^{k}\left(T_{\#}\right) \rightarrow \Omega^{k+1}\left(T_{\#}\right)$ satisfies $\nabla_{\#} \circ \nabla_{\#}=0$, we can define the $k$-th twisted cohomology group by

$$
H^{k}\left(\Omega^{\bullet}\left(T_{\#}\right), \nabla_{\#}\right)=\operatorname{ker}\left(\nabla: \Omega^{k}\left(T_{\#}\right) \rightarrow \Omega^{k+1}\left(T_{\#}\right)\right) / \nabla\left(\Omega^{k-1}\left(T_{\#}\right)\right) .
$$

Let $\mathcal{E}_{c}^{k}\left(T_{\#}\right)$ be the space of smooth $k$-forms on $T_{\#}$ with compact support. By using $\mathcal{E}_{c}^{\bullet}\left(T_{\#}\right)$ and $\nabla_{\#}$, we can also define the twisted cohomology group $H^{k}\left(\mathcal{E}_{c}^{\bullet}\left(T_{\#}\right), \nabla_{\#}\right)$ with compact support.

Fact 3.3 (cf. [1], [3], [13]). Let \# be A or C. Under Assumption 3.1, we have the following.

1. If $k \neq m$, then $H_{k}\left(T_{\#}, U_{\#}\right)=0, H_{k}^{\mathrm{lf}}\left(T_{\#}, U_{\#}\right)=0, H^{k}\left(\Omega^{\bullet}\left(T_{\#}\right), \nabla_{\#}\right)=0$ and $H^{k}\left(\mathcal{E}_{c}^{\bullet}\left(T_{\#}\right), \nabla_{\#}\right)=0$.

2. A canonical map $H_{m}\left(T_{\#}, U_{\#}\right) \rightarrow H_{m}^{\mathrm{lf}}\left(T_{\#}, U_{\#}\right)$ is an isomorphism.

3. There exists an isomorphism $\mathrm{J}: H^{m}\left(\Omega^{\bullet}\left(T_{\#}\right), \nabla_{\#}\right) \rightarrow H^{m}\left(\mathcal{E}_{c}^{\bullet}\left(T_{\#}\right), \nabla_{\#}\right)$.

4. $\operatorname{dim} H_{m}\left(T_{\#}, U_{\#}\right)=\operatorname{dim} H^{m}\left(\Omega^{\bullet}\left(T_{\#}\right), \nabla_{\#}\right)=2^{m}\left(=\operatorname{rank}\right.$ of $\left.E_{\#}\right)$.

\subsection{Intersection pairings}

\subsubsection{Intersection pairing for twisted homology groups}

By replacing $U_{\#}$ with $U_{\#}^{-1}=1 / U_{\#}$, we can also define $H_{m}\left(T_{\#}, U_{\#}^{-1}\right)$ and $H_{m}^{\mathrm{lf}}\left(T_{\#}, U_{\#}^{-1}\right)$. We define the intersection pairing $\mathcal{I}^{h}$ between $H_{m}\left(T_{\#}, U_{\#}\right)$ and $H_{m}^{\mathrm{lf}}\left(T_{\#}, U_{\#}^{-1}\right) \simeq H_{m}\left(T_{\#}, U_{\#}^{-1}\right)$.

Let

$$
\sigma=\sum_{i: \text { fin. }} a_{i} \cdot \Delta_{i} \otimes U_{\#, \Delta_{i}}(t), \quad \tau=\sum_{j} b_{j} \cdot \Delta_{j}^{\prime} \otimes U_{\#, \Delta_{j}^{\prime}}(t)^{-1}
$$

be twisted cycles, where each pair $\left(\Delta_{i}, \Delta_{j}^{\prime}\right)$ does not intersect or intersects transversally. Their intersection number is defined by

$$
\mathcal{I}^{h}(\sigma, \tau)=\sum_{p \in \Delta_{i} \cap \Delta_{j}^{\prime}} a_{i} b_{j} \cdot\left(\Delta_{i}, \Delta_{j}^{\prime}\right)_{p} \cdot U_{\#, \Delta_{i}}(p) \cdot U_{\#, \Delta_{j}^{\prime}}(p)^{-1},
$$

where $\left(\Delta_{i}, \Delta_{j}^{\prime}\right)_{p}$ is the topological intersection number of $\Delta_{i}$ and $\Delta_{j}^{\prime}$ at $p$.

\subsubsection{Intersection pairing for twisted cohomology groups}

We can define the intersection pairing $\mathcal{I}^{c}$ between $H^{m}\left(\Omega^{\bullet}\left(T_{\#}\right), \nabla_{\#}\right)$ and $H^{m}\left(\Omega^{\bullet}\left(T_{\#}\right), \nabla_{\#}^{\vee}\right)$, where $\nabla_{\#}^{\vee}=d-\omega_{\#} \wedge$.

By using the isomorphism $J: H^{m}\left(\Omega^{\bullet}\left(T_{\#}\right), \nabla_{\#}\right) \stackrel{\sim}{\longrightarrow} H^{m}\left(\mathcal{E}_{c}^{\bullet}\left(T_{\#}\right), \nabla_{\#}\right)$ in Fact 3.3 , we can define the intersection number by

$$
\mathcal{I}^{c}(\varphi, \psi)=\int_{T} J(\varphi) \wedge \psi \quad\left(\phi \in H^{m}\left(\Omega^{\bullet}\left(T_{\#}\right), \nabla_{\#}\right), \psi \in H^{m}\left(\Omega^{\bullet}\left(T_{\#}\right), \nabla_{\#}^{\vee}\right)\right) .
$$

Note that we regard $T_{\#} \subset \mathbb{P}^{k}$ as a $2 k$-dimensional real manifold. 


\subsubsection{Twisted period relations}

By using the intersection pairings, we can obtain twisted period relations. In this section, we give only results. For details, see [15].

Let $\left\{\tau_{j}\right\}_{j=1, \ldots, 2^{m}} \subset H_{m}\left(T_{\#}, U_{\#}\right)$ and $\left\{\psi_{j}\right\}_{j=1, \ldots, 2^{m}} \subset H^{m}\left(\Omega^{\bullet}\left(T_{\#}\right), \nabla_{\#}\right)$ be bases. We set

$$
\begin{aligned}
H & =\left(\mathcal{I}^{h}\left(\tau_{i}, \tau_{j}^{\vee}\right)\right)_{i, j=1, \ldots, 2^{m}}, \quad C=\left(\mathcal{I}^{c}\left(\psi_{i}, \psi_{j}\right)\right)_{i, j=1, \ldots, 2^{m}}, \\
\Pi_{+} & =\left(\int_{\tau_{j}} U_{\#} \psi_{i}\right)_{i, j=1, \ldots, 2^{m}}, \quad \Pi_{-}=\left(\int_{\tau_{j}^{\vee}} U_{\#}^{-1} \psi_{i}\right)_{i, j=1, \ldots, 2^{m}},
\end{aligned}
$$

where $\tau_{j}^{\vee} \in H_{m}\left(T_{\#}, U_{\#}^{-1}\right) \simeq H_{m}^{\mathrm{lf}}\left(T_{\#}, U_{\#}^{-1}\right)$ is a twisted cycle defined by the same manner as $\tau_{j}$ with respect to $U_{\#}^{-1}$. Thus, we obtain the twisted period relation

$$
\Pi_{+}{ }^{t} H^{-1}{ }^{t} \Pi_{-}=C \text {. }
$$

Note that the entries of $\Pi_{ \pm}$are hypergeometric integrals.

If the intersection matrix $H$ is diagonal, then its $(i, j)$-entry gives a simple relation:

$$
\sum_{k=1}^{2^{m}} \frac{1}{\mathcal{I}^{h}\left(\tau_{k}, \tau_{k}^{\vee}\right)} \int_{\tau_{k}} U_{\#} \psi_{i} \int_{\tau_{k}^{\vee}} U_{\#}^{-1} \psi_{j}=\mathcal{I}^{c}\left(\psi_{i}, \psi_{j}\right) .
$$

In this article, we construct a basis of the twisted homology group such that the intersection matrix is diagonal, and we rewrite this relation in terms of $F_{A}$ or $F_{C}$.

\section{Twisted cycles and twisted period relations for $F_{A}$ and $F_{C}$}

As mentioned in the previous section, if the homology intersection matrix is diagonal, then we can obtain simple quadratic relations. In fact, cycles corresponding to series solutions (5) and (6) satisfy this property in our cases. In this section, we explain how to construct such cycles and rewrite the twisted period relation (13) in terms of $F_{A}$ or $F_{C}$.

\subsection{Regularization of the $m$-simplex}

Before constructing the twisted cycles for $F_{A}$ and $F_{C}$, we recall the regularization of the $m$ simplex $\Delta^{m}$. The regularization of a locally finite twisted cycle is a twisted cycle that represents the inverse image of the canonical isomorphism $H_{m} \rightarrow H_{m}^{\text {lf }}$ (for $F_{A}$ and $F_{C}$, the isomorphism is mentioned in Fact 3.3). When $m=1$, the regularization of the open interval $\Delta^{1}=(0,1) \subset \mathbb{R}$ is explained in [15]. Similarly to $m=1$, we can construct the regularization of the $m$-simplex for $m \geq 2$.

As an example, we consider the case when $m=2$. We set the multi-valued function $U(t)=$ $t_{1}^{d_{1}} t_{2}^{d_{2}}\left(1-t_{1}-t_{2}\right)^{d_{3}}$ on $T=\mathbb{C}^{2}-\left(\left(t_{1}=0\right) \cup\left(t_{2}=0\right) \cup\left(1-t_{1}-t_{2}=0\right)\right)$. For general cases, refer to [1]. Let us construct the regularization of the locally finite twisted cycle $\Delta^{2} \otimes U \in H_{2}^{\mathrm{lf}}(T, U)$ associated with the 2-simplex

$$
\Delta^{2}=\left\{\left(t_{1}, t_{2}\right) \in \mathbb{R}^{2} \mid t_{1}>0, t_{2}>0,1-t_{1}-t_{2}>0\right\} .
$$


As in the right side of Figure 1, let $\Delta$ be the small triangle included in $\Delta^{2}$ and $I_{i}(i=1,2,3)$ be its boundary. We denote by $S_{i}(i=1,2)$ a positively oriented circle in the $t_{i}$-space starting from the projection of $I_{i}$ to this space and surrounding the divisor $\left(t_{i}=0\right)$, and denote by $S_{3}$ a positively oriented circle with a small radius in the orthogonal complement of the divisor $\left(1-t_{1}-t_{2}=0\right)$ starting from the projection of $I_{3}$ to this space and surrounding the divisor. The twisted cycle $\Delta^{2} \otimes U$ is equal to a finite cycle

$$
\Delta_{\mathrm{reg}}^{2}=\Delta \otimes U+\sum_{i=1}^{3} \frac{\left(S_{i} \times I_{i}\right) \otimes U}{1-\delta_{i}}+\sum_{(i, j)=(1,2),(2,3),(3,1)} \frac{\left(S_{i} \times S_{j}\right) \otimes U}{\left(1-\delta_{i}\right)\left(1-\delta_{j}\right)}
$$

in $H_{2}^{\mathrm{lf}}(T, U)$, where $\delta_{i}=e^{2 \pi \sqrt{-1}} d_{i}$. Thus, $\Delta_{\text {reg }}^{2} \in H_{2}(T, U)$ gives the regularization of $\Delta^{2} \otimes U$.
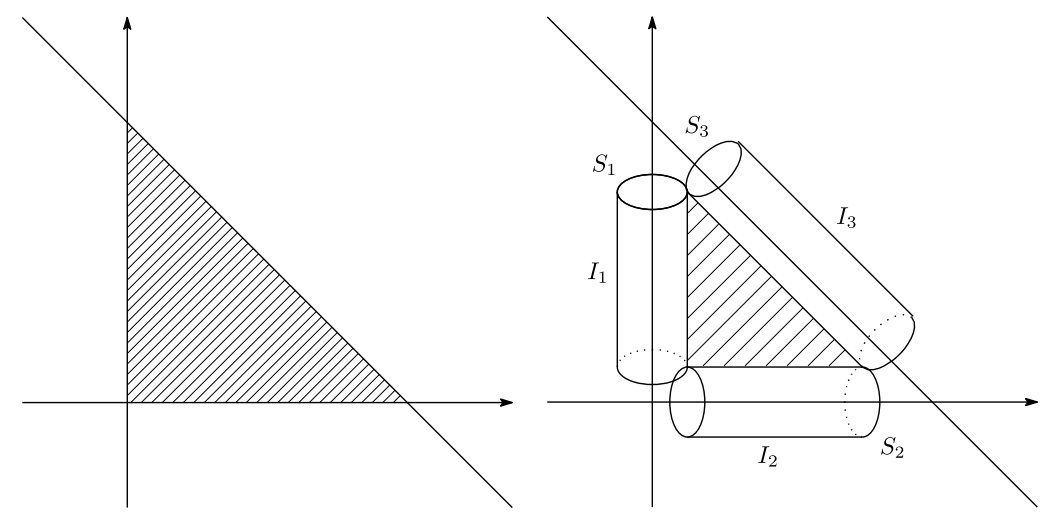

Figure 1: $\Delta^{2}$ and its regularization

\section{2 $F_{C}$}

We construct a basis of the twisted homology group that corresponds to the basis (6) of the solution space of $E_{C}$, which are expressed by $F_{C}$. As an application, we give some quadratic relations between $F_{C}$.

\subsubsection{Twisted cycles corresponding to series solutions}

Assume that $x_{1}, \ldots, x_{m}$ are sufficiently small positive real numbers. We construct a twisted cycle $\Delta_{i_{1} \cdots i_{r}}$ such that the integral $\int_{\Delta_{i_{1} \cdots i_{r}}} U_{C} \frac{d t}{\prod t_{k}\left(1-\sum t_{k}\right)}$ coincides with the series solution

$$
f_{C}^{i_{1} \cdots i_{r}}=\prod_{p=1}^{r} x_{i_{p}}^{1-c_{i_{p}}} \cdot F_{C}\left(a+r-\sum_{p=1}^{r} c_{i_{p}}, b+r-\sum_{p=1}^{r} c_{i_{p}}, c^{I} ; x\right) \quad\left(I=\left\{i_{1}, \ldots, i_{r}\right\} \subset\{1, \ldots, m\}\right),
$$

up to $\Gamma$-factors.

Remark 4.1. Because of $f_{C}^{\emptyset}=F_{C}\left(a, b, c_{1}, \ldots, c_{m} ; x\right), \Delta_{\emptyset}$ is nothing but the twisted cycle $\Delta$ mentioned in Section 2.2.3.

We fix $I=\left\{i_{1}, \ldots, i_{r}\right\} \subset\{1, \ldots, m\}$, and put $\left\{j_{1}, \ldots, j_{m-r}\right\}=\{1, \ldots, m\}-I$. In the discussion below, the index $p$ (resp. $q$ ) runs from 1 to $r$ (resp. from 1 to $m-r$ ). We consider the change of 
variables

$$
t_{i_{p}}=\frac{x_{i_{p}}}{s_{i_{p}}}, \quad t_{j_{q}}=s_{j_{q}}
$$

in the integral

$$
\int \prod t_{k}^{-c_{k}} \cdot\left(1-\sum t_{k}\right)^{\sum c_{k}-a-m}\left(1-\sum \frac{x_{k}}{t_{k}}\right)^{-b} d t
$$

formally. Then we have

$$
\begin{aligned}
& \prod_{p} x_{i_{p}}^{1-c_{i_{p}}} \cdot \int \prod_{p} s_{i_{p}}^{c_{i_{p}}-2} \cdot \prod_{q} s_{j_{q}}^{-c_{j_{q}}} \\
& \cdot\left(1-\sum_{p} \frac{x_{i_{p}}}{s_{i_{p}}}-\sum_{q} s_{j_{q}}\right)^{\sum c_{k}-a-m}\left(1-\sum_{p} s_{i_{p}}-\sum_{q} \frac{x_{j_{q}}}{s_{j_{q}}}\right)^{-b} d s .
\end{aligned}
$$

We set

$$
U_{C, I}(s)=\prod_{p} s_{i_{p}}^{c_{i_{p}}-2} \cdot \prod_{q} s_{j_{q}}^{-c_{j_{q}}} \cdot\left(1-\sum_{p} \frac{x_{i_{p}}}{s_{i_{p}}}-\sum_{q} s_{j_{q}}\right)^{\sum c_{k}-a-m}\left(1-\sum_{p} s_{i_{p}}-\sum_{q} \frac{x_{j_{q}}}{s_{j_{q}}}\right)^{-b} .
$$

If we construct a twisted cycle $\tilde{\Delta}_{i_{1} \cdots i_{r}}$ (in $s$-coordinates) such that

$$
\int_{\tilde{\Delta}_{i_{1} \cdots i_{r}}} U_{C, I}(s) d s=(\text { constant }) \cdot F_{C}\left(a+r-\sum_{p} c_{i_{p}}, b+r-\sum_{p} c_{i_{p}}, c^{I} ; x\right),
$$

then its image $\Delta_{i_{1} \cdots i_{r}} \in H_{m}\left(T_{C}, U_{C}\right)$ (in $t$-coordinates) under the map (14) gives a desired one.

In $\mathbb{R}^{m} \subset \mathbb{C}^{m}$, the set

$$
s_{k}>0,1-\sum \frac{x_{i_{p}}}{s_{i_{p}}}-\sum s_{j_{q}}>0,1-\sum s_{i_{p}}-\sum \frac{x_{j_{q}}}{s_{j_{q}}}>0
$$

is bounded region which includes the direct product

$$
\sigma_{i_{1} \cdots i_{r}}=\left\{\begin{array}{l|l}
\left(s_{1}, \ldots, s_{m}\right) \in \mathbb{R}^{m} & \begin{array}{l}
s_{i_{p}}>\varepsilon, 1-\sum s_{i_{p}}>\varepsilon \\
s_{j_{q}}>\varepsilon, 1-\sum s_{j_{q}}>\varepsilon
\end{array}
\end{array}\right\}
$$

of an $r$-simplex and an $(m-r)$-simplex, for some $\varepsilon>0$. We construct a twisted cycle $\tilde{\Delta}_{i_{1} \cdots i_{r}}$ by using $\sigma_{i_{1} \cdots i_{r}}$ and " $\varepsilon$-neighborhood" of $\left(s_{1}=0\right), \ldots,\left(s_{m}=0\right),\left(1-\sum s_{i_{p}}=0\right),\left(1-\sum s_{j_{q}}=0\right)$.

Example 4.2 (Example for $m=2$ ).

- If $I=\emptyset, \tilde{\Delta}_{\emptyset}=\tilde{\Delta}$ is given as Figure 2. In this case, we have $t_{1}=s_{1}, t_{2}=s_{2}$ and $\tilde{\Delta}=\Delta$. Precisely, it is written as

$$
\begin{aligned}
\Delta & =\sigma \otimes U_{C}+\frac{\left(S_{1} \times I_{1}\right) \otimes U_{C}}{1-\gamma_{1}^{-1}}+\frac{\left(S_{2} \times I_{2}\right) \otimes U_{C}}{1-\gamma_{2}^{-1}}+\frac{\left(S_{3} \times I_{3}\right) \otimes U_{C}}{1-\gamma_{1} \gamma_{2} \alpha^{-1}} \\
& +\frac{\left(S_{1} \times S_{2}\right) \otimes U_{C}}{\left(1-\gamma_{1}^{-1}\right)\left(1-\gamma_{2}^{-1}\right)}+\frac{\left(S_{2} \times S_{3}\right) \otimes U_{C}}{\left(1-\gamma_{2}^{-1}\right)\left(1-\gamma_{1} \gamma_{2} \alpha^{-1}\right)}+\frac{\left(S_{3} \times S_{1}\right) \otimes U_{C}}{\left(1-\gamma_{1} \gamma_{2} \alpha^{-1}\right)\left(1-\gamma_{1}^{-1}\right)},
\end{aligned}
$$

where $\alpha=e^{2 \pi \sqrt{-1} a}, \gamma_{k}=e^{2 \pi \sqrt{-1}} c_{k}$ and the radius of the circle $S_{i}$ is $\varepsilon$. 


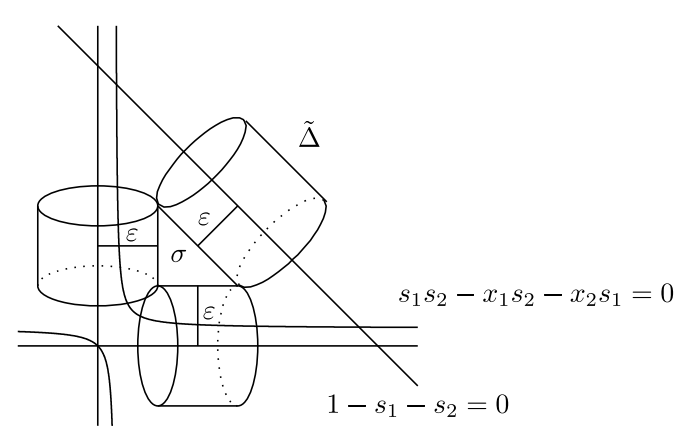

Figure 2: $\tilde{\Delta}$ for $m=2$

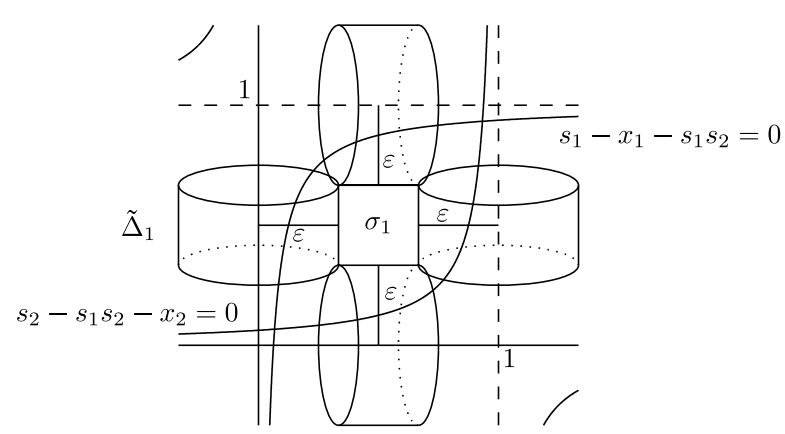

Figure 3: $\tilde{\Delta}_{1}$ for $m=2$

- If $I=\{1\}, \tilde{\Delta}_{1}$ is given as Figure 3 .

In any cases, some circles may surround two divisors.

Proposition 4.3 ([3]).

$$
\begin{gathered}
\int_{\tilde{\Delta}_{i_{1} \cdots i_{r}}} U_{C, I}(s) d s=\frac{\prod_{p} \Gamma\left(c_{i_{p}}-1\right) \cdot \prod_{q} \Gamma\left(1-c_{j_{q}}\right) \cdot \Gamma\left(\sum c_{k}-a-m+1\right) \Gamma(1-b)}{\Gamma\left(\sum_{p} c_{i_{p}}-a-r+1\right) \Gamma\left(\sum_{p} c_{i_{p}}-b-r+1\right)} \\
\cdot F_{C}\left(a+r-\sum_{p} c_{i_{p}}, b+r-\sum_{p} c_{i_{p}}, c^{I} ; x\right) .
\end{gathered}
$$

Proof. Consider the power series expansion of the left-hand side with respect to $x_{1}, \ldots, x_{m}$. By our construction, this expansion converges uniformly. As a coefficient of $x_{1}^{n_{1}} \cdots x_{m}^{n_{m}}$ in the power series expansion, the integral

$$
\int_{\tilde{\Delta}_{i_{1} \cdots i_{r}}} \prod_{p} s_{i_{p}}^{c_{i_{p}}-n_{i_{p}}-2} \prod_{q} s_{j_{q}}^{-c_{j_{q}}-n_{j_{q}}} \cdot\left(1-\sum_{p} s_{i_{p}}\right)^{-b-\sum n_{j_{q}}}\left(1-\sum_{q} s_{j_{q}}\right)^{\sum c_{k}-a-m-\sum n_{i_{p}}} d s
$$

appears. If we regard $\tilde{\Delta}_{i_{1} \cdots i_{r}}$ as a twisted cycle loading this integrand, each circle in $\tilde{\Delta}_{i_{1} \cdots i_{r}}$ surrounds only one divisor. Thus, $\tilde{\Delta}_{i_{1} \cdots i_{r}}$ is nothing but the regularization of the direct product of two simplices $\Delta^{r}$ (in $\left(s_{i}\right)_{i \in I^{-}}$-coordinates) and $\Delta^{m-r}$ (in $\left(s_{j}\right)_{j \in J}$-coordinates). The integral (15) is equal to

$$
\begin{aligned}
& \int_{\Delta^{r}} \prod_{p} s_{i_{p}}^{c_{i_{p}}-n_{i_{p}}-2}\left(1-\sum_{p} s_{i_{p}}\right)^{-b-\sum n_{j_{q}}} d s_{I} \cdot \int_{\Delta^{m-r}} \prod_{q} s_{j_{q}}^{-c_{j_{q}}-n_{j_{q}}}\left(1-\sum_{q} s_{j_{q}}\right)^{\sum c_{k}-a-m-\sum n_{i_{p}}} d s_{I c} \\
& =\frac{\prod_{p} \Gamma\left(c_{i_{p}}-n_{i_{p}}-1\right) \cdot \Gamma\left(-b-\sum_{q} n_{j_{q}}+1\right)}{\Gamma\left(-b+\sum_{p} c_{i_{p}}-\sum n_{k}-r+1\right)} \cdot \frac{\prod_{q} \Gamma\left(-c_{j_{q}}-n_{j_{q}}+1\right) \cdot \Gamma\left(\sum c_{k}-a-m-\sum_{p} n_{i_{p}}+1\right)}{\Gamma\left(\sum_{p} c_{i_{p}}-a-\sum n_{k}-r+1\right)},
\end{aligned}
$$

where $d s_{I}=d s_{i_{1}} \wedge \cdots \wedge d s_{i_{r}}, d s_{I^{c}}=d s_{j_{1}} \wedge \cdots \wedge d s_{j_{m-r}}$. By using $\Gamma(z) \Gamma(1-z)=\pi / \sin (\pi z)$ and $(a)_{n}=\Gamma(a+n) / \Gamma(a)$, we obtain the proposition.

Therefore, we obtain a twisted cycle corresponding to the solution $f_{C}^{i_{1} \cdots i_{r}}$. 
Theorem 4.4 ([3]). Let $\Delta_{i_{1} \cdots i_{r}}$ be the twisted cycle in $T_{C}$ which is obtained as the image of $\tilde{\Delta}_{i_{1} \cdots i_{r}}$ under the correspondence $t_{i_{p}}=\frac{x_{i_{p}}}{s_{i_{p}}}, t_{j_{q}}=s_{j_{q}}$. Then we have

$$
\begin{aligned}
& \int_{\Delta_{i_{1} \cdots i_{r}}} \prod t_{k}^{-c_{k}} \cdot\left(1-\sum t_{k}\right)^{\sum c_{k}-a-m} \cdot\left(1-\sum \frac{x_{k}}{t_{k}}\right)^{-b} d t \\
& =\frac{\prod_{p} \Gamma\left(c_{i_{p}}-1\right) \cdot \prod_{q} \Gamma\left(1-c_{j_{q}}\right) \cdot \Gamma\left(\sum c_{k}-a-m+1\right) \Gamma(1-b)}{\Gamma\left(\sum_{p} c_{i_{p}}-a-r+1\right) \Gamma\left(\sum_{p} c_{i_{p}}-b-r+1\right)} \cdot f_{C}^{i_{1} \cdots i_{r}} .
\end{aligned}
$$

Proof. Consider the change of variables (14), and use Proposition 4.3.

Theorem 4.5 ([3]). We put $\alpha=e^{2 \pi \sqrt{-1} a}, \beta=e^{2 \pi \sqrt{-1} b}, \gamma_{k}=e^{2 \pi \sqrt{-1} c_{k}}$.

1. $I \neq I^{\prime} \Longrightarrow I^{h}\left(\Delta_{I}, \Delta_{I^{\prime}}^{\vee}\right)=0$;

2. $I^{h}\left(\Delta_{i_{1} \cdots i_{r}}, \Delta_{i_{1} \cdots i_{r}}^{\vee}\right)=(-1)^{r} \cdot \frac{\prod_{q} \gamma_{j_{q}} \cdot\left(\alpha-\prod_{p} \gamma_{i_{p}}\right)\left(\beta-\prod_{p} \gamma_{i_{p}}\right)}{\prod_{k}\left(\gamma_{k}-1\right) \cdot\left(\alpha-\prod_{k} \gamma_{k}\right)(\beta-1)}$.

Sketch of the proof. 1 . Since the function $f_{C}^{I}$ corresponding to $\Delta_{I}$ has the same monodromy property as $\prod_{i \in I} x_{i}^{1-c_{i}}$ around $x=(0, \ldots, 0)$, the claim follows from the monodromy invariance of $I^{h}$.

2. The self-intersection number of $\Delta_{i_{1} \cdots i_{r}}$ coincides with that of $\tilde{\Delta}_{i_{1} \cdots i_{r}}$. By using the results in [9], we can evaluate it.

\subsubsection{Cohomology intersection numbers}

Except for $m=2$ (Appell's $F_{4}$ ), we do not have so many results for intersection numbers of the twisted cohomology groups.

Theorem 4.6 ([3]). We put

$$
\varphi=\frac{d t_{1} \wedge \cdots \wedge d t_{m}}{\prod_{k} t_{k} \cdot\left(1-\sum_{k} t_{k}\right)}, \quad \varphi^{\prime}=\frac{d t_{1} \wedge \cdots \wedge d t_{m}}{\prod t_{k} \cdot\left(1-\sum t_{k}\right) \cdot\left(1-\sum \frac{x_{k}}{t_{k}}\right)} .
$$

Then we have

$$
\begin{aligned}
& \mathcal{I}^{c}\left(\varphi, \varphi^{\prime}\right)=0, \\
& \mathcal{I}^{c}(\varphi, \varphi)=(2 \pi \sqrt{-1})^{m}\left(\frac{1}{\sum c_{k}-a-m+1}+\frac{1}{b+m-\sum c_{k}}\right) \sum_{\left\{I^{(r)}\right\}} \prod_{r=1}^{m-1} \frac{1}{b+r-\sum c_{i_{p}^{(r)}}},
\end{aligned}
$$

where $\left\{I^{(r)}\right\}$ is a sequence of subsets of $\{1, \ldots, m\}$ such that

$$
\{1, \ldots, m\} \supsetneq I^{(m-1)} \supsetneq \cdots \supsetneq I^{(2)} \supsetneq I^{(1)} \neq \emptyset,
$$

and we write $I^{(r)}=\left\{i_{1}^{(r)}, \ldots, i_{r}^{(r)}\right\}$.

Roughly speaking, intersection numbers are evaluated as

$$
\sum_{P: \text { intersection point of } m \text { divisors }} \operatorname{Res}_{t=P}(\text { some differential form }),
$$

if the pole divisor of $\omega_{C}=d \log U_{C}$ are normally crossing ([12]). In this case, we need to blow up $\mathbb{C}^{m}$. For detailed calculations, refer to [3]. 


\subsubsection{Twisted period relations for $F_{C}$}

Since the intersection matrix $H$ with respect to the basis $\left\{\Delta_{I}\right\}_{I} \subset H_{m}\left(T_{C}, U_{C}\right)$ is diagonal by Theorem 4.5, we obtain twisted period relations (13) as follows:

$$
\begin{aligned}
\mathcal{I}^{c}\left(\varphi, \varphi^{\prime}\right) & =\sum_{I} \frac{1}{\mathcal{I}^{h}\left(\Delta_{i_{1} \cdots i_{r}}, \Delta_{i_{1} \cdots i_{r}}^{\vee}\right)} \cdot \int_{\Delta_{i_{1} \ldots i_{r}}} U_{C} \varphi \cdot \int_{\Delta_{i_{1} \ldots i_{r}}} U_{C}^{-1} \varphi^{\prime}, \\
\mathcal{I}^{c}(\varphi, \varphi) & =\sum_{I} \frac{1}{\mathcal{I}^{h}\left(\Delta_{i_{1} \cdots i_{r}}, \Delta_{i_{1} \cdots i_{r}}^{\vee}\right)} \cdot \int_{\Delta_{i_{1} \ldots i_{r}}} U_{C} \varphi \cdot \int_{\Delta_{i_{1} \ldots i_{r}}^{\vee}} U_{C}^{-1} \varphi .
\end{aligned}
$$

By Theorem 4.4, these integrals are expressed by $F_{C}$. Thus, we obtain two quadratic relations

$$
0=\sum_{I}(-1)^{r}\left(a_{i_{1} \cdots i_{r}}-1\right) \cdot F_{C}\left(a_{i_{1} \cdots i_{r}}, b_{i_{1} \cdots i_{r}}, c^{i_{1} \cdots i_{r}} ; x\right) \cdot F_{C}\left(2-a_{i_{1} \cdots i_{r}}, 1-b_{i_{1} \cdots i_{r}}, \check{c}^{i_{1} \cdots i_{r}} ; x\right),
$$

and

$$
\begin{aligned}
& \frac{(1-a+b) \cdot \prod\left(1-c_{k}\right)}{b b_{1} \cdots m} \cdot \sum_{\left\{I^{(r)}\right\}} \prod_{r=1}^{m-1} \frac{1}{b_{I^{(r)}}} \\
& =\sum_{I}(-1)^{r} \frac{1-a_{i_{1} \cdots i_{r}}}{b_{i_{1} \cdots i_{r}}} \cdot F_{C}\left(a_{i_{1} \cdots i_{r}}, b_{i_{1} \cdots i_{r}}, c^{i_{1} \cdots i_{r}} ; x\right) \cdot F_{C}\left(2-a_{i_{1} \cdots i_{r}},-b_{i_{1} \cdots i_{r}}, \check{c}^{i_{1} \cdots i_{r}} ; x\right),
\end{aligned}
$$

where we put

$$
a_{i_{1} \cdots i_{r}}=a+r-\sum c_{i_{p}}, \quad b_{i_{1} \cdots i_{r}}=b+r-\sum c_{i_{p}}, \quad \check{c}^{i_{1} \cdots i_{r}}=(2, \ldots, 2)-c^{i_{1} \cdots i_{r}} .
$$

\subsubsection{Twisted period relations for Appell's $F_{4}(m=2)$}

In the case of $m=2$, we have more results. In [6], we put

$$
\begin{aligned}
\varphi_{1} & =\frac{d t_{1} \wedge d t_{2}}{t_{1} t_{2}\left(1-t_{1}-t_{2}\right)}, \quad \varphi_{2}=\frac{d t_{1} \wedge d t_{2}}{t_{2}\left(1-t_{1}-t_{2}\right)}, \\
\varphi_{3} & =\frac{d t_{1} \wedge d t_{2}}{t_{1}\left(1-t_{1}-t_{2}\right)}, \quad \varphi_{4}=\frac{d t_{1} \wedge d t_{2}}{\left(1-t_{1}-t_{2}\right)\left(t_{1} t_{2}-t_{2} x_{1}-t_{1} x_{2}\right)}
\end{aligned}
$$

and evaluate their intersection matrix. For example,

Theorem 4.7 ([6]).

$$
\mathcal{I}^{c}\left(\varphi_{4}, \varphi_{4}\right)=\frac{2 \cdot(2 \pi \sqrt{-1})^{2}}{\left(c_{1}+c_{2}-a-2\right) \cdot(-b)} \cdot \frac{1}{x_{1}^{2}+x_{2}^{2}-2 x_{1} x_{2}-2 x_{1}-2 x_{2}+1} .
$$

Note that $x_{1}^{2}+x_{2}^{2}-2 x_{1} x_{2}-2 x_{1}-2 x_{2}+1$ is a factor of the defining polynomial of the singular locus $S_{C}$ (see Section 2.3.3). By using this intersection number, we obtain the following relation:

$$
\begin{aligned}
& b(a-1) F_{4}\left(a, b+1, c_{1}, c_{2} ; x\right) \cdot F_{4}\left(2-a, 1-b, 2-c_{1}, 2-c_{2} ; x\right) \\
& -b_{1}\left(a_{1}-1\right) F_{4}\left(a_{1}, b_{1}+1,2-c_{1}, c_{2} ; x\right) \cdot F_{4}\left(2-a_{1}, 1-b_{1}, c_{1}, 2-c_{2} ; x\right) \\
& -b_{2}\left(a_{2}-1\right) F_{4}\left(a_{2}, b_{2}+1, c_{1}, 2-c_{2} ; x\right) \cdot F_{4}\left(2-a_{2}, 1-b_{2}, 2-c_{1}, c_{2} ; x\right) \\
& +b_{12}\left(a_{12}-1\right) F_{4}\left(a_{12}, b_{12}+1,2-c_{1}, 2-c_{2} ; x\right) \cdot F_{4}\left(2-a_{12}, 1-b_{12}, c_{1}, c_{2} ; x\right) \\
& =\frac{2\left(1-c_{1}\right)\left(1-c_{2}\right)}{x_{1}^{2}+x_{2}^{2}-2 x_{1} x_{2}-2 x_{1}-2 x_{2}+1} .
\end{aligned}
$$


4.3 $F_{A}$

We can apply a similar argument for $F_{A}$. We introduce some results, and omit detailed calculations.

\subsubsection{Twisted cycles corresponding to series solutions}

For $I=\left\{i_{1}, \ldots, i_{r}\right\} \subset\{1, \ldots, m\}$, we put $\left\{j_{1}, \ldots, j_{m-r}\right\}=\{1, \ldots, m\}-I$. We consider the change of variables

$$
t_{i_{p}}=\frac{s_{i_{p}}}{x_{i_{p}}}, \quad t_{j_{q}}=s_{j_{q}}
$$

in the integral (11), formally. Similarly to Section 4.2.1, we can construct a twisted cycle $\tilde{\Delta}_{i_{1} \cdots i_{r}}$ (in $s$-coordinates) such that

$$
\begin{aligned}
& \int_{\tilde{\Delta}_{i_{1} \cdots i_{r}}} \prod_{p} s_{i_{p}}^{c_{i_{p}}-2}\left(1-\frac{x_{i_{p}}}{s_{i_{p}}}\right)^{c_{i_{p}}-b_{i_{p}}-1} \cdot\left(1-\sum_{p} s_{i_{p}}-\sum_{q} x_{j_{q}} s_{j_{q}}\right)^{-a} \cdot \prod_{q} s_{j_{q}}^{b_{j_{q}}-1}\left(1-s_{j_{q}}\right)^{c_{j_{q}}-b_{j_{q}}-1} d s \\
& =\text { (constant) }) \cdot F_{A}\left(a+r-\sum_{p} c_{i_{p}}, b^{I}, c^{I} ; x\right) .
\end{aligned}
$$

Then, we obtain the following theorems.

Theorem 4.8 ([4]). We can construct twisted cycles $\Delta_{i_{1} \cdots i_{r}} \in H_{m}\left(T_{A}, U_{A}\right)$ corresponding to the series solutions:

$$
\begin{aligned}
& \int_{\Delta_{i_{1} \cdots i_{r}}} \prod\left(t_{k}^{b_{k}} \cdot\left(1-t_{k}\right)^{c_{k}-b_{k}-1}\right) \cdot\left(1-\sum x_{k} t_{k}\right)^{-a} d t \\
& =e^{\pi \sqrt{-1}\left(\sum b_{i_{p}}-\sum c_{i_{p}}+r\right)} \cdot \frac{\Gamma(1-a) \prod_{p} \Gamma\left(c_{i_{p}}-1\right)}{\Gamma\left(\sum_{p} c_{i_{p}}-a-r+1\right)} \cdot \prod_{q} \frac{\Gamma\left(b_{j_{q}}\right) \Gamma\left(c_{j_{q}}-b_{j_{q}}\right)}{\Gamma\left(c_{j_{q}}\right)} \cdot f_{A}^{i_{1} \cdots i_{r}} .
\end{aligned}
$$

Theorem 4.9 ([4]). We put $\alpha=e^{2 \pi \sqrt{-1} a}, \beta_{k}=e^{2 \pi \sqrt{-1} b_{k}}, \gamma_{k}=e^{2 \pi \sqrt{-1} c_{k}}$.

1. $I \neq I^{\prime} \Longrightarrow I^{h}\left(\Delta_{I}, \Delta_{I^{\prime}}^{\vee}\right)=0$;

2. $I^{h}\left(\Delta_{i_{1} \cdots i_{r}}, \Delta_{i_{1} \cdots i_{r}}^{\vee}\right)=\frac{\alpha-\prod_{p} \gamma_{i_{p}}}{(\alpha-1) \prod_{p}\left(1-\gamma_{i_{p}}\right)} \cdot \prod_{q} \frac{\beta_{j_{q}}\left(1-\gamma_{j_{q}}\right)}{\left(1-\beta_{j_{q}}\right)\left(\beta_{j_{q}}-\gamma_{j_{q}}\right)}$.

Remark 4.10. In [11], our construction of twisted cycles corresponding to series solutions is generalized to a regular holonomic GKZ-hypergeometric system ( $A$-hypergeometric system) which is associated with an integer matrix $A$. When $A$ has a unimodular triangulation, their intersection numbers have been also obtained in [11]. In the other cases, the intersection numbers are studied in [5].

\subsubsection{Cohomology intersection numbers}

In [13], the intersection matrix with respect to the basis

$$
\varphi_{i_{1} \cdots i_{r}}=\frac{d t_{1} \wedge \cdots \wedge d t_{m}}{\prod_{p}\left(t_{i_{p}}-1\right) \cdot \prod_{q} t_{j_{q}}} \quad\left(\begin{array}{l}
I=\left\{i_{1}, \ldots, i_{r}\right\} \subset\{1, \ldots, m\}, \\
\left\{j_{1}, \ldots, j_{m-r}\right\}=\{1, \ldots, m\}-I
\end{array}\right)
$$


of the twisted cohomology group $H^{m}\left(\Omega^{\bullet}\left(T_{A}\right), \nabla_{A}\right)$ is evaluated.

For $I=\left\{i_{1}, \ldots, i_{r}\right\} \subset\{1, \ldots, m\}$, we put

$$
A_{i_{1} \cdots i_{r}}=A_{I}=\sum_{\left\{I^{(l)}\right\}} \prod_{l=1}^{r} \frac{1}{a-\sum c_{i_{p}^{(l)}}+l},
$$

where $\left\{I^{(l)}\right\}$ is a sequence of subsets of $I$ such that

$$
I=I^{(r)} \supsetneq I^{(r-1)} \supsetneq \cdots \supsetneq I^{(2)} \supsetneq I^{(1)} \neq \emptyset .
$$

Fact 4.11 ([13]). We have

$$
\mathcal{I}^{c}\left(\varphi_{I}, \varphi_{I^{\prime}}\right)=(2 \pi \sqrt{-1})^{m} \cdot \sum_{N \subset\{1, \ldots, m\}}\left(A_{N} \prod_{n \notin N} \frac{\delta_{I, I^{\prime}}(n)}{\tilde{b}_{I, I^{\prime}}(n)}\right),
$$

where

$$
\delta_{I, I^{\prime}}(n)=\left\{\begin{array}{ll}
1 & \left(n \in\left(I \cap I^{\prime}\right) \cup\left(I^{c} \cap I^{c}\right)\right), \\
0 & \text { (otherwise) },
\end{array} \quad \tilde{b}_{I, I^{\prime}}(n)=\left\{\begin{array}{l}
c_{n}-1-b_{n}\left(n \in I \cap I^{\prime}\right), \\
b_{n}\left(n \in I^{c} \cap I^{\prime c}\right) .
\end{array}\right.\right.
$$

\subsubsection{Twisted period relations for $F_{A}$}

We write one example of quadratic relations. The twisted period relation (13) with respect to $\mathcal{I}^{c}\left(\varphi_{\emptyset}, \varphi_{12 \cdots m}\right)$ and the basis $\left\{\Delta_{I}\right\} \subset H_{m}\left(T_{A}, U_{A}\right)$ is reduced into the relation

$$
\frac{\prod\left(1-c_{k}\right)}{a} \cdot \sum_{\left\{I^{(l)}\right\}} \prod_{l=1}^{r} \frac{1}{a-\sum_{p} c_{i_{p}^{(l)}}+l}=\sum_{I} \frac{(-1)^{r}}{a_{I}} \cdot F_{A}\left(a_{I}, b^{I}, c^{I} ; x\right) \cdot F_{A}\left(-a_{I}, \check{b}^{I}, \check{c}^{I} ; x\right),
$$

where a sequence $\left\{I^{(l)}\right\}$ is same as Section 4.2.2, and we put

$$
\begin{aligned}
& a_{I}=a_{i_{1} \cdots i_{r}}=a+r-\sum_{p} c_{i_{p}}, \\
& \left\{\begin{array}{l}
b_{i_{p}}^{I}=b_{i_{p}}+1-c_{i_{p}}, \quad\left\{\begin{array}{l}
c_{i_{p}}^{I}=2-c_{i_{p}}, \\
b_{j}^{I}=b_{j},
\end{array} c_{j}\right. \\
\check{b}^{I}=(1, \ldots, 1)-b^{I}, \quad \check{c}^{I}=(2, \ldots, 2)-c^{I} .
\end{array}\right.
\end{aligned}
$$

\section{Acknowledgments}

This article is based on the talk in the meeting at the University of Padova in September 2019. The author thanks to Professor Pierpaolo Mastrolia for his invitation. The author also grateful to Hjalte Frellesvig, Federico Gasparotto, Manoj K. Mandal and Luca Mattiazzi for having attended the meeting, and for having looked after him so well during his stay in Padova.

Finally, the author expresses his gratitude to the speakers of the work shop "MathemAmplitudes 2019: Intersection Theory \& Feynman Integrals" for accepting that the proceedings include his contribution nevertheless he could not participate the work shop. 


\section{References}

[1] K. Aomoto and M. Kita, translated by K. Iohara, Theory of Hypergeometric Functions Springer Verlag, New York, 2011.

[2] K. Cho and K. Matsumoto, Intersection theory for twisted cohomologies and twisted Riemann's period relations I, Nagoya Math. J. 139 (1995) 67-86.

[3] Y. Goto, Twisted cycles and twisted period relations for Lauricella's hypergeometric function $F_{C}$, Internat. J. Math. 24 (2013) 1350094 19pp.

[4] Y. Goto, Twisted period relations for Lauricella's hypergeometric functions $F_{A}$, Osaka J. Math. 52 (2015) 861-877.

[5] Y. Goto and S.-J. Matsubara-Heo, Homology and cohomology intersection numbers of GKZ systems, arXiv:2006.07848.

[6] Y. Goto and K. Matsumoto, The monodromy representation and twisted period relations for Appell's hypergeometric function $F_{4}$, Nagoya Math. J. 217 (2015) 61-94.

[7] R. Hattori and N. Takayama, The singular locus of Lauricella's $F_{C}$, J. Math. Soc. Japan 66 (2014) 981-995.

[8] M. Kita, On Hypergeometric Functions in Several Variables 1. New integral representations of Euler type, Japan. J. of Math. 18 (1992), 25-74.

[9] M. Kita and M. Yoshida, Intersection theory for twisted cycles I, II, Math. Nachr. 166 (1994), 287-304, 168 (1994), 171-190.

[10] G. Lauricella, Sulle funzioni ipergeometriche a più variabili, Rend. Circ. Mat. Palermo 7 (1893) 111-158.

[11] S.-J. Matsubara-Heo, Euler and Laplace integral representations of GKZ hypergeometric function, arXiv:1904.00565.

[12] K. Matsumoto, Intersection numbers for logarithmic k-forms, Osaka J. Math. 35 (1998) 873-893.

[13] K. Matsumoto, Pfaffian of Lauricella's hypergeometric system $F_{A}$, Rokko Lectures in Mathematics 24 (2016), 23-37.

[14] K. Matsumoto, Appell and Lauricella hypergeometric functions, to appear in Chapter 3 of Encyclopedia of Special Functions: The Askey-Bateman Project Volume 2. Multivariable Special Functions.

[15] K. Matsumoto, Introduction to the Intersection Theory for Twisted Homology and Cohomology Groups, in proceedings of MathemAmplitudes 2019: Intersection Theory \& Feynman Integrals (MA2019).

[16] N. E. Steenrod, Homology with local coefficients, Ann. of Math. (2) 44 (1943) 610-627. 